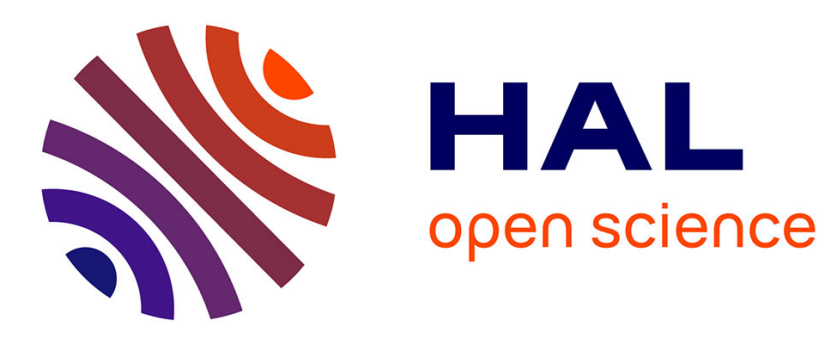

\title{
Saturated structured confocal microscopy with theoretically unlimited resolution
}

\author{
Olivier Haeberlé, Bertrand Simon
}

\section{To cite this version:}

Olivier Haeberlé, Bertrand Simon. Saturated structured confocal microscopy with theoretically unlimited resolution. Optics Communications, 2009, 282 (18), pp.3657-3664. 10.1016/j.optcom.2009.06.025 . hal-00865488

\section{HAL Id: hal-00865488 \\ https://hal.science/hal-00865488}

Submitted on 24 Sep 2013

HAL is a multi-disciplinary open access archive for the deposit and dissemination of scientific research documents, whether they are published or not. The documents may come from teaching and research institutions in France or abroad, or from public or private research centers.
L'archive ouverte pluridisciplinaire HAL, est destinée au dépôt et à la diffusion de documents scientifiques de niveau recherche, publiés ou non, émanant des établissements d'enseignement et de recherche français ou étrangers, des laboratoires publics ou privés. 


\title{
Saturated structured confocal microscopy with theoretically unlimited resolution
}

\author{
Olivier Haeberlé and Bertrand Simon \\ Laboratoire MIPS EA2332, Université de Haute-Alsace IUT Mulhouse, 61 rue A. Camus \\ F-68093 Mulhouse Cedex France \\ olivier.haeberle@uha.fr, bertrand.simon@uha.fr
}

The resolution of fluorescence microscopes is limited by diffraction, which determines the extension of their point spread functions. We propose and study numerically a simple method, based on a combination of subtraction microscopy with regular and annular excitation beams, which permits to double the resolution compared to wide-field microscopy. When combined with the fluorescence saturation phenomenon, this approach would be able to deliver a resolution of a few tens of nanometers.

PACS numbers: $\quad$ 07.60.Pb, 87.64.mk, 87.57.cf, 42.30.Va, Keywords: point spread function engineering, fluorescence microscopy, image formation 


\section{Introduction}

The fluorescence microscope is the instrument of choice when studying living cells and tissues. Recent developments have permitted to overcome the conventional resolution limit with the inventions of the confocal, theta, SPIM and 4Pi microscopes for example [1-4]. The better resolution for these instruments is obtained by improving the illumination or the detection process or both, but fundamentally, these instruments are still diffraction limited.

In order to further improve the resolution, non-linear phenomena have to be involved. For example, STED microscopy takes profit of quantum effects to inhibit the fluorescence process at undesired locations, therefore efficiently reducing the actual size of the point spread function (PSF). A resolution about $100 \mathrm{~nm}$ in the three dimensions has been obtained [5], and for 2-D surface studies, resolution as small as $40 \mathrm{~nm}$ has been proven [5].

Another possible approach is to make use of the saturation effect when exciting the specimen. In the general case, saturation has to be avoided, as it is known to indeed degrade the resolution. When cleverly combined with a properly structured illumination, saturation may however be used to improve the resolution [7-8]. With this method, particles as small as $50 \mathrm{~nm}$ have been imaged [7]. The dynamic nature of saturation may also be taken into account in order to break the Abbe limit [9].

A third technique consists in switching from a resolution problem, where one aims at distinguishing small features, which emit light simultaneously, to a localization problem, where those same features emit light sequentially. Known as PALM, F-PALM, STORM, or PALMIRA [10-13], this technique has proven to deliver a nanometric resolution in far-field fluorescence microscopy. 
These techniques have proven an unsurpassed resolution, and are still constantly being refined and improved, in terms of ease of use, speed and precision/resolution. One may however notice some possible limitations. For example, up to know, and even in growing numbers, yet few fluorophores (compared to the very large variety of fluorophores available for fluorescence microscopy) do resist to the excitation/stimulated process involved in STED microscopy. Localization techniques involve bleaching of the fluorophores, which is unfavorable for dynamics imaging, or use photoswitchable fluorophores, which are not yet available in a great variety, and require a large number of images to be processed, so that speed may be a limitation for imaging live specimens. It therefore still appears of interest to go on looking for other possible approaches, which may permit to obtain a high resolution in fluorescence microscopy, and may be complementary to these already well established techniques.

We propose and study theoretically a method, which combines a very simple image processing technique, namely subtraction microscopy involving only two images [14], with structured saturation excitation, which in principle may be used with a larger number of fluorophores. A possible domain of application of this approach could be the study of 2-D specimens, as for example cell membranes, DNA fragments or microtubules deposited on a glass slide.

\section{Structured subtraction microscopy}

Subtraction microscopy has been proposed to improve fluorescence microscopy imaging properties, by combining images taken in wide-field microscopy and confocal microscopy. The method we propose is essentially a variant of subtraction microscopy in which the confocal acquisition is modified. 
In order to model the wide-field PSF (as well as the detection PSF $_{\text {det }}$ in confocal setup), we use the model of Haeberlé [15], considering a fluorescent dipole of moment $\mathbf{p}_{\mathbf{e}}$ induced by the excitation. The electric field being collected by the objective, and refocused onto the detector is given by:

$$
\begin{aligned}
\mathrm{E}_{x^{\prime}} & =p_{e x}^{*}\left(I_{0 \mathrm{det}}+I_{2 \mathrm{det}} \cos 2 \phi_{d}\right)+p_{e y}^{*}\left(I_{2 \mathrm{det}} \sin 2 \phi_{d}\right) \\
& -2 i I_{1 \mathrm{det}} p_{e z}^{*} \cos \phi_{d} \\
\mathrm{E}_{y^{\prime}} & =p_{e x}^{*} I_{2 \operatorname{det}} \sin 2 \phi_{d}+p_{e y}^{*}\left(I_{0 \mathrm{det}}-I_{2 \mathrm{det}} \cos 2 \phi_{d}\right) \\
& -2 i I_{1 \mathrm{det}} p_{e z}^{*} \sin \phi_{d}
\end{aligned}
$$

where $\phi_{d}$ represents the polar angle for the considered point of the detector, with respect to the optical axis. For detailed explanations about the computation of the diffraction integrals $I_{n d e t}$, the interested reader is referred to Ref. [15-18], which .give a complete description of the considered configuration. For the sake of simplicity, we consider in the following a simple model with rotating free molecules and that the fluorescence mechanism implies randomly polarized emission, so that angular dependences vanishes and the PSF is circularly symmetric and given by:

$$
P S F_{w f}(r, z=0)=\left|I_{0 \operatorname{det}}\right|^{2}+2\left|I_{1 \operatorname{det}}\right|^{2}+\left|I_{2 \operatorname{det}}\right|^{2}
$$

$\mathrm{r}$ being the radial distance from the optical axis. As we consider in this study only very shallow, 2-D like specimens, the PSF is computed in the focal plane, so that the elevation $\mathrm{z}$ is set to be zero.. In conventional subtraction microscopy, a beam splitter is introduced within the detection path of a confocal microscope, and two detectors are used, one with a tightly closed pinhole for confocal detection, and one without pinhole for wide field imaging. Alternatively, one can record successively to images, by widely opening the pinhole to mimic wide field microscopy. 
The first improvement we propose is to replace conventional confocal excitation (with a circularly or randomly polarized laser so as to consider a circularly symmetric illumination PSF), by a structured illumination using an azimuthally polarized laser. In that case, the illumination PSF is given by [19]:

$$
\operatorname{PSF}_{\text {ill-az }}(r, z=0)=\left|I_{\text {iill-az }}\right|^{2}
$$

with:

$$
I_{1 i l l-a z}=\int_{0}^{\alpha} \cos ^{1 / 2} \theta \sin \theta J_{1}(k r \sin \theta) \sin \theta d \theta
$$

Figure 1 displays $\mathrm{PSF}_{\mathrm{wf}}$ for wide field microscopy, $\mathrm{PSF}_{\text {conf }}$ for confocal microscopy, the illumination $\mathrm{PSF}_{\text {ill-az }}$ with an azimuthally polarized laser beam, the confocal $\mathrm{PSF}_{\text {conf-az }}$, obtained by multiplying the former by the latter, and the final subtraction microscopy PSF $_{\text {sub-az. }}$. For these computations, we consider a N.A. $=1.4$ oil immersion objective, an excitation performed at $400 \mathrm{~nm}$, and a detection at $450 \mathrm{~nm}$, corresponding to the use of Cascade Blue dye (Molecular Probes). The final $\mathrm{PSF}_{\text {sub-az }}$ in subtraction microscopy is defined by:

$$
P S F_{\text {sub-az }}=P S F_{w f}-\alpha P S F_{\text {conf }-a z}
$$

The wide field PSF $_{w f}$ presents a full width at half maximum (FWHM) of $190 \mathrm{~nm}$ (Fig 1(a)). The illumination PSF $_{\text {ill-az }}$ presents a hollow structure with a FWHM of $104 \mathrm{~nm}$, but the total FWHM is $326 \mathrm{~nm}$ (Fig. 1(b)). When confocalized, the central hole as a smaller FWHM of $76 \mathrm{~nm}$, but the total FWHM is still $250 \mathrm{~nm}$ (Fig. 1(c)). This is much wider than the wide field $\mathrm{PSF}_{\mathrm{wf}}$, and will preclude an efficient reduction of the final PSF $_{\text {sub-az }}$ width. Indeed, as the hollow structure being 
present in the illumination $\mathrm{PSF}_{\text {ill-az }}$ is subtracted from the wide field $\mathrm{PSF}_{\mathrm{wf}}$, one can interpret it as

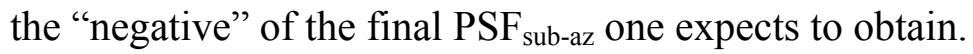

Contrary to conventional subtraction microscopy, which takes benefit of the difference in slopes between a conventional and a narrower confocal PSF, we would like here to directly suppress the most external part of the wide field $\mathrm{PSF}_{\mathrm{wf}}$ with the confocal $\mathrm{PSF}_{\text {conf-az. }}$ We must therefore adapt the wide field $\mathrm{PSF}_{\mathrm{wf}}$ to the confocal one, which is in our configuration and contrary to the usual case wider. This may be performed simply by reducing the numerical aperture in the wide field mode. Obviously, the key-point of subtraction microscopy is the proper adjustment of the parameter $\alpha$, in order to benefit from the best resolution improvement, but avoiding the apparition of negative regions in the final $\mathrm{PSF}_{\text {sub-az }}$ (or alternatively in the final image obtained from the subtraction of the confocal image from the wide field image). We here would like to recall that, in the proposed configuration, one subtracts a confocal PSF from a wide field one. As a consequence, the apparition of negative regions is mainly excluded because the confocal PSF falls down faster that the wide field PSF. This is the opposite of the "classical" subtraction microscopy, where one subtracts a wide field PSF from a confocal one, and therefore negative regions may rapidly appear, because of the larger lateral extension of the wide field PSF with respect to the confocal one.

Both parameters (N.A. and $\alpha$ ) must be adjusted in order to obtain a substantial improvement of the resolution. Figure $1(\mathrm{~d})$ is computed with NA $=1.1$ (solid line in Fig. 1(d)) for wide field detection, and with $\alpha=2 / 3$.

The final $\mathrm{PSF}_{\text {sub-az }}$ has a FWHM of $90 \mathrm{~nm}$ only, to be compared with the theoretical lateral resolution of $190 \mathrm{~nm}$ for the wide field microscope (as computed from a high NA vectorial model [20], and not from the more commonly used $\mathrm{R}_{\text {Abbe }}=\lambda /(2 \mathrm{~N} . \mathrm{A})=.161 \mathrm{~nm}$, which 
overestimates the resolution when considering the FWHM of the PSF.). For the ideal usual confocal microscope, a $130 \mathrm{~nm}$ resolution is predicted (see Fig. 1(a)).

The configuration we propose may therefore permit to obtain a $35 \%$ resolution improvement compared to a confocal microscope, or a factor 2 compared to wide field microscopy. We obtain the same gain in resolution than with confocal fluorescence microscopy using laterally interfering excitation beams [21]. However, the final resolution is now isotropic with only two images, while this other method further needs recombination of several images (typically 4) in order to obtain an isotropic resolution [22]. Furthermore, producing an azimuthally polarized illumination beam from a linearly polarized beam seems to be easier than having three focused beam interfering at the focal point as proposed in Ref. [21]. Note that we find the same factor 2 as in wide-field structured illumination fluorescence microscopy [23]. In the next section, we shall show how this technique of subtraction microscopy, when combined with fluorescence saturation, may be used to even further improve the resolution.

\section{Saturated structured subtraction microscopy}

Fluorescence saturation was proposed to obtain a theoretically unlimited resolution with patterned excitation wide-field microscopy. Gustafsson [7] demonstrated a 2-D resolution of $50 \mathrm{~nm}$ with this technique. Heintzmann et al. [8] showed that a similar resolution may be obtained if using a two-dimensionally structured excitation pattern. It was shown that in principle, an unlimited resolution might be obtained with fluorescence saturation techniques, as long as the considered fluorophore can sustain saturation without being photobleached. 
We shall now show how the configuration we propose may attain similar performances by also making use of the fluorescence saturation phenomenon. For the sake of simplicity, we use the same model as Heintzmann et al. $[8,24]$ to describe the fluorescence saturation phenomenon:

$$
I_{e m}(x, y)=\frac{S^{-1} I_{e x c}(x, y)}{S^{-1}+I_{e x c}(x, y)} \rho(x, y)
$$

Because the fluorescence intensity $I_{\mathrm{em}}$ remains a linear process with the fluorophore density $\rho$, even with saturation, one can define the emittability [21] $\mathrm{PSF}_{\mathrm{em}}$ of the system as:

$$
P S F_{e m}(x, y)=\frac{S^{-1} P S F_{i l l}(x, y)}{S^{-1}+P S F_{i l l}(x, y)}
$$

And for the confocal case, $\mathrm{PSF}_{\mathrm{conf}}=\mathrm{PSF}_{\mathrm{em}} \mathrm{PSF}_{\text {det. }}$. More elaborate models may be used [7,9], but should not change the general conclusions, as mentioned by Heintzmann et al. [8,24]. Figures 2 display the results obtained for increasing $\mathrm{S}$ ( $\mathrm{S}=0$ corresponding to the linear regime). Figure 2(a) shows the emittability PSF $_{\text {em. }}$. One can note that, as expected, each lobe increases in extension when increasing S. Figure 2(b) shows the corresponding confocal configurations: indeed, confocalization suppresses the growing outer secondary lobes, but the most interesting feature is that the central hole is thinner increasing $\mathrm{S}$. As a consequence, $\mathrm{PSF}_{\text {sub-az-sat }}$ for subtraction microscopy with fluorescence saturation is narrower and narrower as shown by Fig. 2(c).

However, in our case, because PSF $_{\text {conf-az-sat }}$ changes (Fig. 2(b)), the subtraction coefficient $\alpha$ must be adapted for each saturation level. We used $\alpha=0.72-0.74-0.81-0.85-0.90$ for $\mathrm{S}=1-2-5-10-25$, respectively. The FWHM of the final PSF $_{\text {sub-az-sat }}$ is 70-60-45-35-24 nm, respectively. Note however that an optimal value of the parameter $\alpha$ is not mandatory to obtain an improved 
resolution. Figure 2(d) shows $\mathrm{PSF}_{\text {sub-az-sat }}$ computed for $\mathrm{S}=5$ and $\alpha=0.81$ (optimal value) and $\alpha=0.75$. The resolution is only degraded from $45 \mathrm{~nm}$ to $48 \mathrm{~nm}$. The image would however present a lower contrast, because of the higher lateral extensions of the final PSF.

In principle, this resolution may be arbitrarily improved by increasing S, provided of course that suitable fluorophores that may sustain the process without being bleached are available and that the noise level in the recorded images is low enough. Gustafsson suggested possible approaches in Ref. [7]. But even using lower saturation levels as in Refs [7,8,24] may still permit to obtain a resolution in the $50 \mathrm{~nm}$ range, much better that regular confocal imaging and without the requirement of high-speed lasers and instrumentation as in STED microscopy, and with very simple image processing.

Up to now, we used in order to demonstrate the principle of the method we propose, an ideal case with an infinitely small detection pinhole and no noise. Fluorescence confocal microscopy is often characterized by a rather low signal to noise ration, and the detection pinhole shall be open to get a useful signal. In the following, we study the influence of the pinhole size and noise onto the performances of saturated structured subtraction microscopy, considering a saturation factor $S=5$.

The equation describing the PSF formation in confocal microscopy shall be written in the more general case as:

$$
P S F_{\text {conf }}=P S F_{\text {exc }} \times\left[P S F_{d e t} \otimes \text { pinhole }\right]
$$

where the second terms describes the effect of scanning through a finite size pinhole as a convolution of the ideal detection PSF by the pinhole geometry. Figures 3 describe the effect of the pinhole size in saturated structured subtraction microscopy, for circular pinholes of 
increasing diameter. The diameter of the pinhole is given as a function of the Airy Disk (AD) size, taken as $1.22 \lambda / \mathrm{NA}$, and for the configuration we consider (with $\lambda=450 \mathrm{~nm}$ for detection and $\mathrm{NA}=1.4$ ) we obtain $392 \mathrm{~nm}$, approximated to $400 \mathrm{~nm}$ in the next. The upper figure described the effect onto the detection $\mathrm{PSF}_{\text {det. }}$ For small pinhole diameters $(100 \mathrm{~nm}=1 / 4 \mathrm{AD}$ and $200 \mathrm{~nm}=1 / 2 \mathrm{AD}$ ), the influence is negligeable. But as soon as the pinhole is open wider, the detection PSF widens very fast. The influence of this varying PSF $_{\text {det }}$ onto the confocal PSF can be noticed on the left-column graphs, depicting PSF $_{\text {conf-az-sat }}$ as a function of the pinhole size, the theoretical ideal case (infinitely small pinhole) being recalled for the sake of comparison on each image. Note the rapid widening of the lobes with increasing pinhole size. For the larger pinhole sizes ( $1 \mathrm{AD}$ and 1.25 $\mathrm{AD}$ ), note also the reemergence of the secondary lobes of the excitation $\mathrm{PSF}_{\text {exc-az, }}$ which are not anymore suppressed by the confocalization process.

The consequence of this widening of the main lobes, and the reemergence of the secondary lobes can be seen on the left-column graphs of Fig. 3. They depict the final PSF $_{\text {sub-az-sat }}$ obtained by adjusting the subtraction parameter $\alpha$ to each case. Because of the wider lobes, one has in fact to decrease $\alpha$ when opening the pinhole, in order to avoid the apparition of negative regions in the final PSF. As a consequence, the possible resolution decreases very fast, and one obtains a FWHM of the final PSF $\mathrm{Pub}_{\text {suz-sat }}$ of 46-55-80-130-150 nm with a pinhole diameter of $1 / 4,1 / 2,3 / 4$, 1 and $5 / 4$ of the Airy Disk, and with a subtraction parameter $\alpha=0.80,0.73,0.55,0.33$ and 0.25 , respectively. It then appears that using narrow pinholes (up to 3/4 AD) is mandatory in order to keep the high resolution, which would be allowed by the use of a saturated azimuthally polarized excitation. Note that this need for narrow pinhole is a common feature to subtraction microscopy in general: for example, in Heintzmann et al. [25] the pinhole size is $0.32,0.43$ or 0.5 Airy Unit only. 
This mandatory use of small pinhole sizes in order to provide an efficient improvement of the resolution is clearly detrimental in terms of signal to noise ratio. In the following, we therefore study the influence of the noise level onto the performances of the proposed approach. We keep now constant saturation factor $S=5$ and pinhole size $=1 / 2$ Airy Disk, and vary the noise level. Figures 4 shows the results obtained assuming pixels of $10 \mathrm{~nm}$ (necessary to well describe a PSF with FWHM of only $55 \mathrm{~nm}$ ), and a maximum photon statistics of $10^{4}, 5.10^{3}, 10^{3}, 5.10^{2}$, and $10^{2}$ photons for the pixel of maximum intensity for both the widefield and confocal PSF to be substracted. One can notice the rapid degradation of the shapes of the PSFs with increasing noise. As a consequence, in order to avoid the appearance of negative regions, one has to again tweek the subtraction coefficient $\alpha$ to the noise level. In order to estimate the possible resolution, we adopt the following procedure: for each photon statistics, 10 simulations for $\operatorname{PSF}_{\mathrm{wf}}$ and $\mathrm{PSF}_{\text {conf-az-sat }}$ have been performed. For each case, the subtraction parameter $\alpha$ is recomputed so as to get a final $\mathrm{PSF}_{\text {sub-az-sat }}$ free of negative regions. Then, the lowest subtraction parameter $\alpha$ is selected. Doing so, we get the most conservative estimation of the final resolution, as we have seen before that lowering $\alpha$ degrades the resolution. We finally obtain an estimated resolution of 58-60-65-70 and $85 \mathrm{~nm}$ for the $10^{4}, 5.10^{3}, 10^{3}, 5.10^{2}$, and $10^{2}$ photons at maximum cases, respectively, and with computed $\alpha=0.69,0.66,0.61,0.55$ and 0.45 , respectively. These values are to be compared with $55 \mathrm{~nm}$ FWHM resolution with $\alpha=0.73$ for the ideal, noise-free case.

Note that the influence of the noise onto the resolution is less severe than the influence of the pinhole size. A simple attempt explanation to this a priori surprising fact can be the following: at the maximum of the wide field $\mathrm{PSF}_{\mathrm{wf}}$, one indeed subtracts a zero of $\mathrm{PSF}_{\text {conf-az-sat, }}$, which is favorable in terms of keeping a better signal to noise ratio (and therefore final resolution) in the final PSF $_{\text {sub-az-sat. }}$ This is in contrary to the more conventional subtraction microscopy, where 
both wide field and confocal PSFs have their maxima located along the optical axis: subtracting both signals will greatly diminish the signal to noise ratio of the final PSF, which is detrimental for keeping a high resolution.

In order to illustrate the better imaging capabilities of the method we propose, we show on Fig. 5 a simulation for a 2-D object; which depicts the Abbe formula (Fig. 5(a)). Figure 5(b) shows the simulated image for wide field imaging. Note the important blurring. Figure 5(c) shows the confocal image with azimuthal excitation with a saturation factor $\mathrm{S}=5$, and confocal detection with a pinhole size of $1 / 2$ Airy Disk (for the sake of simplicity, no noise is considered now). The use of a donut shaped PSF renders it difficult to interpret: for example, vertical or horizontal details appear doubled as can be seen inspecting the Greek letter $\lambda$ or the fraction bar. Finally, Figure 5(d) shows the subtraction microscopy image: A very noticeable improvement in the image quality appears, the formula being now clearly readable. The graph shows the profiles, along the line indicated by the arrows, of the original object, and of the simulated images of this object in wide-field microscopy and saturated subtraction microscopy. It clearly illustrates the better resolution provided by the latter, which exhibits correctly the three peaks corresponding to the bottom of the greek letter $\lambda$, while the lower resolution of wide field microscopy would not permit to discriminate them.

\section{Conclusion and perspectives}

In conclusion, we have proposed a method, which shall permit to obtain a very high resolution in fluorescence microscopy. The key points are the production of an excitation PSF with a central hole, which may be obtained by using azimuthally polarized beams, and use of fluorescence saturation as in Refs. [7,8]. Our results confirm those of Schwartz and Oron [27], who proposed 
another theoretical approach combining fluorescence saturation and pupil filters to modify the excitation PSF.

The method we propose relies on a double imaging approach. While acquiring the first image with wide-field illumination and linear excitation is standard, the second image to be subtracted supposes fluorescence saturation. Such a process is known to induce photobleaching and phototoxicity, which would preclude dynamic or live imaging for example. This point was discusses by Heintzmann [24] and Heintzmann et al. [8], who concluded that: "photobleaching will be more severe than in the linear methods only in cases where excited (singlet or triplet) state absorption plays a major role" (from ref. [24]). Similarly, Gustafsson notes when proposing saturated structured illumination microscopy that: "Highly photostable fluorescent labels do exist, including several types of nanoparticles. Diamond nanocrystals containing nitrogen-vacancy color centers, for example, are known to fluoresce under continuous saturation conditions for hours without bleaching." (from ref. [7]). The limitations for saturated structured confocal microscopy are therefore probably the same than for saturated structured illumination microscopy [7] or saturated patterned excitation microscopy $[8,24]$ and obviously, care must be taken in the fluorophore choice. Note however that even without considering saturation, a noticeable gain in resolution is possible, as shown by Fig. 1.

One must notice however two things. First, the final image remains blurred by a halo surrounding the formula. This halo is the result of the remaining side-lobes visible on the final PSF $_{\text {sub-az-sat }}$ (see for example Figure 3). Second, one can notice that Figure 5(c) seems to already deliver more details, even if hardly interpretable. For example, the Greek letter $\lambda$ is more visible (even if doubled) than on Figure 5(b). These indeed indicate that the subtraction process, while very simple to understand the gain in resolution, may not be optimal in terms of image processing. As an alternative, the two required images, which are recorded under different 
blurring conditions, may be recombined by weighted averaging [25] or Fourier fusion techniques. An inverse filter corresponding to the annular PSF may even permit to reduce the number of images to be taken to only one. Inverse filtering is however sensitive to noise, while multikernel deconvolution permits to even further improve the resolution, and has been proven to be very robust to both noise and kernel estimation errors [22].

The method we have proposed here is presently restricted to 2-D specimens: the possibility of reducing the 3-D focal spot using such a simple technique would also be of great usefulness, for example for cell imaging. Such a possibility is conditioned to the realization of a narrow 3-D dark focal spot, similarly to the optimization, which has already been proposed for RESOLFT microscopy [26]. Note that the requirements may however be much more stringent, as subtraction microscopy involves a linear process, while RESOLF techniques are highly nonlinear.

The subtraction process is sensitive to the relative sizes of the linear wide-field PSF and the saturated structured confocal PSF, as shown by the study of the influence of the pinhole size onto the resolution. As a consequence, a potential issue is the possible presence of aberrations because of a mismatch between the immersion oil, and the observed specimen. Spherical aberrations can lead to a significant decrease of the resolution for both wide-field and confocal techniques. With oil immersion objectives, one should then restrict the application of such methods to very flat specimens only, as for example cell membrane studies, for which the observation plane corresponds to the surface of the coverglass, where the objective is designed to work with a minimum of aberrations. To minimize the negative influence of aberrations, the use of water immersion objectives with $\mathrm{NA}=1.2$ would constitute a good solution. As the various PSFs involved in the approach we propose directly scales with the numerical aperture (for both linear 
and saturated PSFs), the results we computed for $\mathrm{NA}=1.4$ can be directly translated for water immersion objectives. For example, Figure 1(d) for water immersion objective should be computed with $\mathrm{NA}=1.2$ for the confocal azimuthal excitation, $\mathrm{NA}=1.1 *(1.2 / 1.4)$ for the widefield detection, which would lead with the same factor $2 / 3$ for the subtraction process to a final resolution of $90 *(1.4 / 1.2)=105 \mathrm{~nm}$.

Then, we should mention another approach using fluorescence saturation in order to improve the resolution. The excitation intensity is modulated at a frequency $\omega$, and the fluorescence signal is demodulated at higher-order harmonic frequencies $(2 \omega, 3 \omega, \ldots)$, which leads to a narrower higher-order harmonic PSF than the regular PSF. The authors of $[27,28]$ have already proven an improved experimental resolution in a biological sample with this technique, which, (together with the Gustafsson's results [7]), demonstrate the validity of the concept of using saturation in order to improve the resolution in fluorescence microscopy.

Finally, we would like to emphasize the relative simplicity of the experiment we propose, which could be based on a modified confocal microscope. The first image would simply be taken with a very large pinhole (or without any pinhole), which would then produce the same image as a wide-field microscope and with a standard laser beam illumination. The key point of the proposed approach is then production of the azimuthally polarized beam for recording the second image. Devices such as those proposed in Refs. [30-32] could be inserted into the illumination arm of the confocal set-up in order to produce the desired polarization state required for recording the second image. We believe that such a rather simple implementation may attract the attention of experimentalists interested in high-resolution fluorescence imaging. 


\section{Acknowledgements}

The authors gratefully thank the anonymous referee for his/her valuable comments and suggestions. 


\section{References}

1. D. Minsky, "Memoir on Inventing the Confocal Scanning Microscope" Scanning 10, pp. $128-138(1988)$

2. E. H. K. Stelzer and S. Lindek, "Fundamental reduction of the observation volume in farfield light microscopy by detection orthogonal to the illumination axis: confocal theta microscopy” Opt. Commun. 111, pp. 536 -547 (1994)

3. C.J. Engelbrecht and E.H.K Stelzer, "Resolution enhancement in a light-sheet-based microscope (SPIM)” Opt. Lett. 31, pp.1477-1479 (2006)

4. S. Hell and E.H.K. Stelzer, "Fundamental improvement of resolution with a 4Pi-confocal fluorescence microscope using two-photon excitation” Opt. Comm. 93, pp.277-282 (1992)

5. T. A. Klar et al., "Fluorescence microscopy with diffraction resolution barrier broken by stimulated emission ” Proc. Natl. Acad. Sci. USA 97, pp. 8206-8210 (2000)

6. K. I. Willig et al., "STED microscopy resolves nanoparticle assemblies” New J. Phys. 8-106 (2006)

7. M. G. L. Gustafsson, "Nonlinear structured-illumination microscopy: Wide-field fluorescence imaging with theoretically unlimited resolution” Proc. Natl. Acad. Sci. USA 102, pp. 13081-13086(2005)

8. R. Heintzmann, T. M. Jovin and C. Cremer, "Saturated patterned excitation microscopy-a concept for optical resolution improvement” J. Opt. Soc. Am. A 19, pp. 1599-1609 (2002)

9. J. Enderlein, "Breaking the diffraction limit with dynamic saturation optical microscopy" Appl. Phys. Let. 87, 094105 (2005)

10. E. Betzig et al., "Imaging Intracellular Fluorescent Proteins at Nanometer Resolution" Science 313, pp. 1642-1645 (2006) 
11. S. T. Hess, T. P. K. Girirajan,yz and M. D. Mason, "Ultra-High Resolution Imaging by Fluorescence Photoactivation Localization Microscopy (FPALM)" Biophys. J. 91, pp. 4258$4272(2006)$

12. . M. J. Rust, M. Bates and X. Zhuang, "Sub-diffraction-limit imaging by stochastic optical reconstruction microscopy (STORM)" Nature Meth. 3, pp. 793-796 (2006)

13. A. Egner, et al., "Fluorescence nanoscopy in whole cells by asynchronous localization of photoswitching emitters”. Biophys. J. 93, 3285 - 3290 (2007)

14. S.J. Hewlett and T. Wilson, "Resolution enhancement in three-dimensional confocal microscopy. Mach”. Vis. Appl. 4, pp. 233-242 (1991)

15. O. Haeberlé, "Focusing of light through a stratified medium: a practical approach for computing microscope point spread functions: Part II: confocal and multiphoton microscopy" Opt. Comm. 235, pp. 1-10 (2004)

16. O. Haeberlé, et al. "The point spread function of optical microscopes imaging through stratified media" Opt. Exp. 11, 2964-2969 (2003)

17. 5. P. Török, P.D. Higdon, and T. Wilson, "On the general properties of polarized light conventional and confocal microscopes," Opt. Comm. 148, 300-315 (1998)

18. P. Török, "Propagation of electromagnetic dipole waves through dielectric interfaces," Opt. Lett. 25, 1463-1465 (2000)

19. K. S. Youngworth and T. G. Brown, "Focusing of high numerical aperture cylindrical-vector beams" Opt. Exp. 7, pp. 77-87 (2000)

20. O. Haeberlé, "Focusing of light through a stratified medium: a practical approach for computing microscope point spread functions. Part I: Conventional microscopy” Opt. Comm. 216, pp. 55-63 (2003) 
21. O. Haeberlé and B. Simon, "Improving the lateral resolution in confocal fluorescence microscopy using laterally interfering excitation beams" Opt. Comm. 259, pp. 400-408 (2006)

22. B. Simon and O. Haeberlé, "Multi-kernel deconvolution applied to confocal fluorescence microscopy with engineered point spread function” J. Eur. Opt. Soc.-RP, 1-06028 (2006)

23. M. G. L. Gustafsson, "Surpassing the lateral resolution limit by a factor of two using structured illumination microscopy" J. Microsc. 198, pp. 82-87 (2000)

24. R. Heintzmann, "Saturated patterned excitation microscopy with two-dimensional excitation patterns" Micron 34, pp. 283-291 (2003)

25. R. Heintzmann, et al., "Resolution enhancement by subtraction of confocal signals taken at different pinhole sizes" Micron 34, pp. 293-300 (2003)

26. J. Keller, A. Schönle and S. W. Hell "Efficient fluorescence inhibition patterns for RESOLFT microscopy”,. Opt. Expr. 15, pp. 3361-3371 (2007)

27. O. Schwartz and D. Oron, "Using variable pupil filters to optimize the resolution in multiphoton and saturable fluorescence confocal microscopy, " Opt. Lett. 34, 464-466 (2009)

28. K. Fujita, et.al., "High-Resolution Confocal Microscopy by Saturated Excitation of Fluorescence" Phys. Rev. Lett. 99, 228105 (2007)

29. M. Yamanaka, et.al., "Beyond the diffraction-limit biological imaging by saturated excitation microscopy" J. Biomed. Opt. 13, 050507 (2008)

30. T. Grosjean, A. Sabac and D. Courjon, "A versatile and stable device allowing the efficient generation of beams with radial, azimuthal or hybrid polarizations", Opt. Comm. 252, pp. 12$21(2005)$ 
31. K. J. Moh, et al., "Generating radial or azimuthal polarization by axial sampling of circularly polarized vortex beams" Appl. Opt. 46, pp. 7544-7551 (2007)

32. A. I. Konoiko and S. N. Zhdanovich, "Formation of radial and azimuthal polarization of light”, J. Opt. Techn., 74, pp. 356-358 (2007) 


\section{FIGURE CAPTIONS}

Fig. 1: (a): wide-field $\mathrm{PSF}_{\mathrm{wf}}$ and confocal PSF for randomly polarized illumination (b): excitation PSF $_{\text {ill-az }}$ for azimuthally polarized illumination. (c): confocal hollow PSF $_{\text {conf-az. }}$ (d): subtraction microscopy PSF. $\lambda_{\text {exc }}=400 \mathrm{~nm}, \lambda_{\text {det }}=450 \mathrm{~nm}$. NA $=1.4$ except for solid curve in (d) whith N.A.=1.1.

Fig. 2: (a): Emittability PSFs for various saturation levels. (b): Corresponding confocalized PSFs. (c): resulting PSFs for subtraction microscopy. (d): subtraction PSFs for $\mathrm{S}=5$ and $\alpha=0.81$ (solid line) and $\alpha=0.75$ (dashed line). . $\lambda_{\text {exc }}=400 \mathrm{~nm}, \lambda_{\text {det }}=450 \mathrm{~nm}$. NA $=1.4$ except for solid curve in (d) whith N.A. $=1.1$.

Fig. 3: Influence of the pinhole size on the final resolution. Top: detection PSF for increasing pinhole size: 100, 200, 300, 400 and $500 \mathrm{~nm}(1 / 4,1 / 2,3 / 4,1$ and 5/4 of the Airy Disk, respectively). Left column: influence on the azimuthally polarized confocalized PSF (thin solid line: ideal case with infinitely small pinhole) for increasing pinhole size. Right column: final subtraction microscopy PSF for increasing pinhole size. In all cases: saturation factor $\mathrm{S}=5$ other parameters are the same as for Figs. 1 and 2.

Fig. 4: Noise influence on the final resolution for saturation factor $S=5$ and a pinhole size of $200 \mathrm{~nm}\left(1 / 2\right.$ Airy Disk) for decreasing photon statistics: no noise, $10^{4}, 5.10^{3}, 10^{3}, 5.10^{2}$, and $10^{2}$ photons at the maximum of the PSFs, respectively. Other parameters are the same as for Figs. 3.

Fig. 5: 2D simulations of subtraction microscopy with saturation factor $\mathrm{S}=5$, and a pinhole size of $200 \mathrm{~nm}$ (1/2 Airy Disk) with no noise, same other parameters as for Fig. 1. (a): numerical object under consideration. (b): regular wide field image. (c): azimuthally polarized confocalized image. (d): subtration of (c) from (b). Note the improved image quality, which is attested by the plot of the profiles along the line indicated by the arrows for the original object, the wide field microscopy image and the saturated structured confocal microscopy image. 


\section{FIGURE 1}
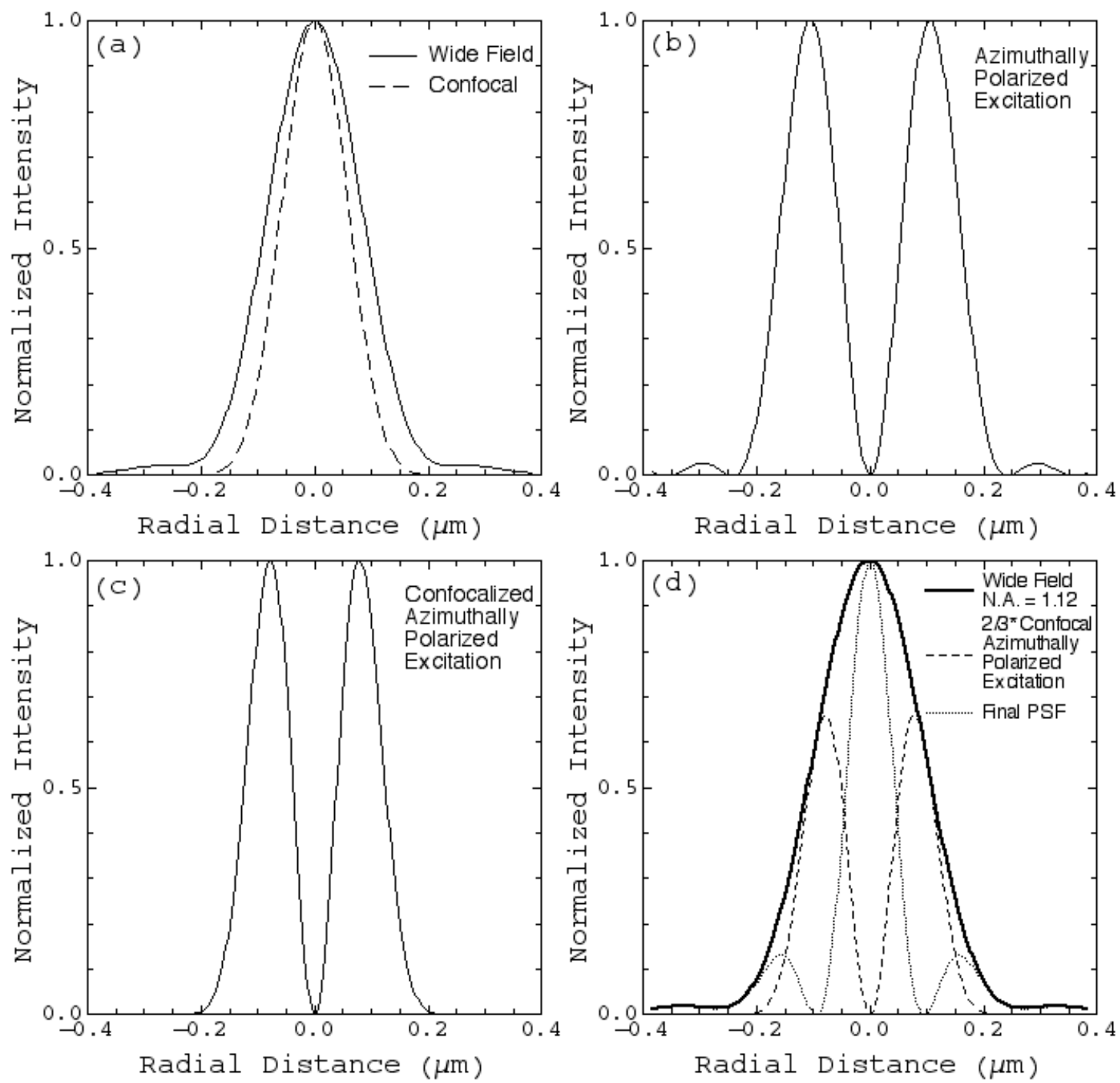
FIGURE 2
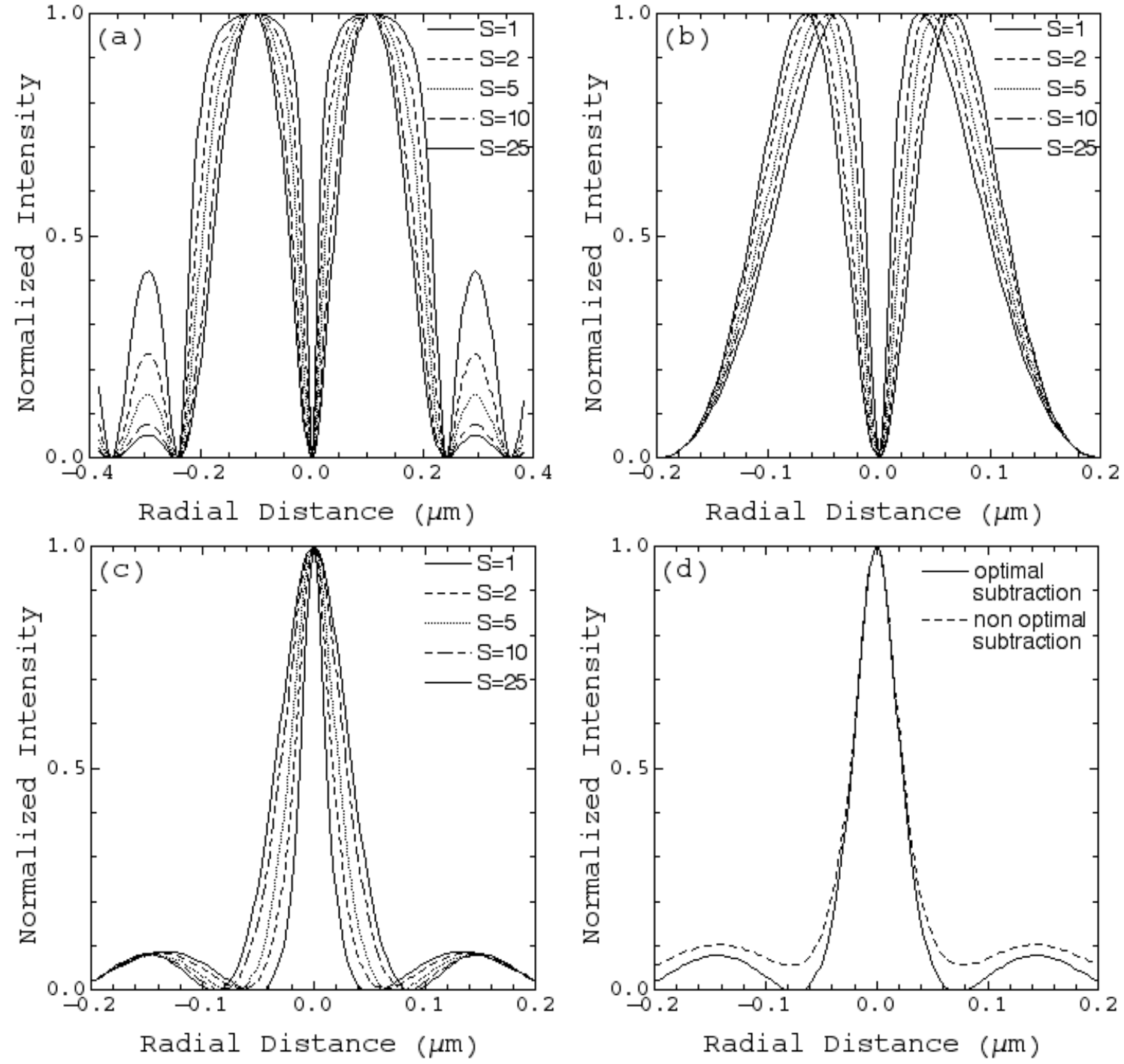
FIGURE 3
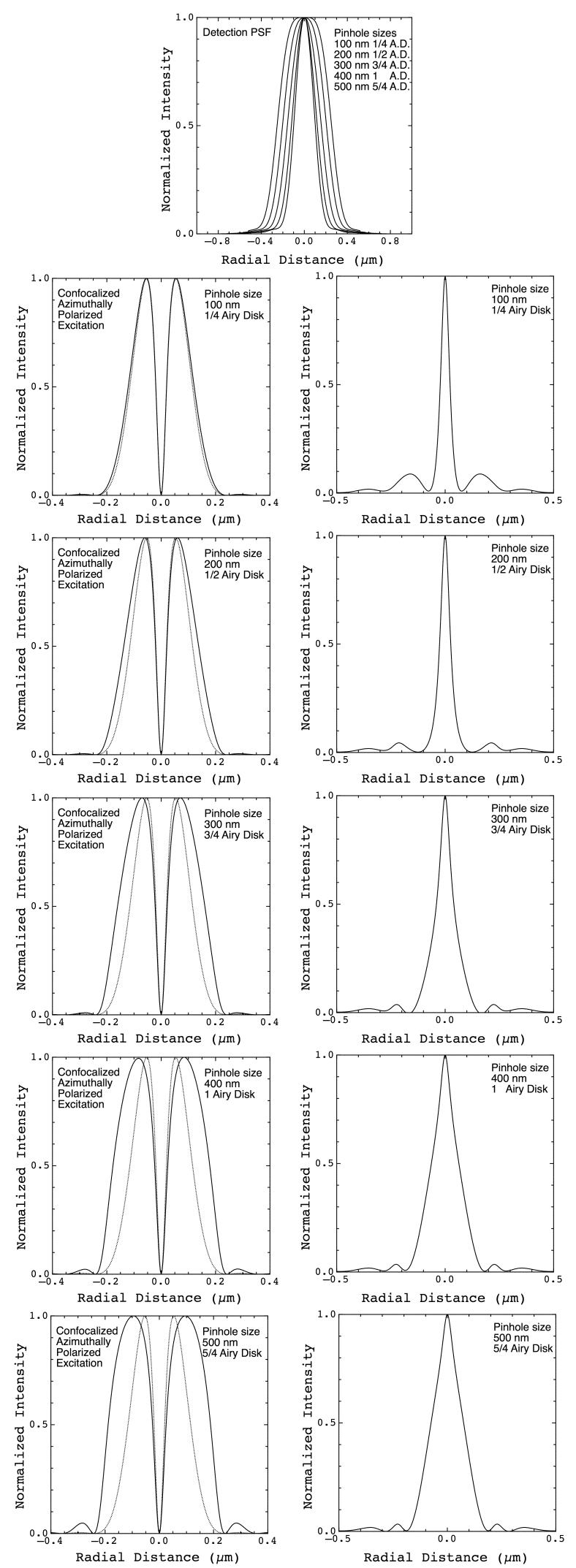
FIGURE 4
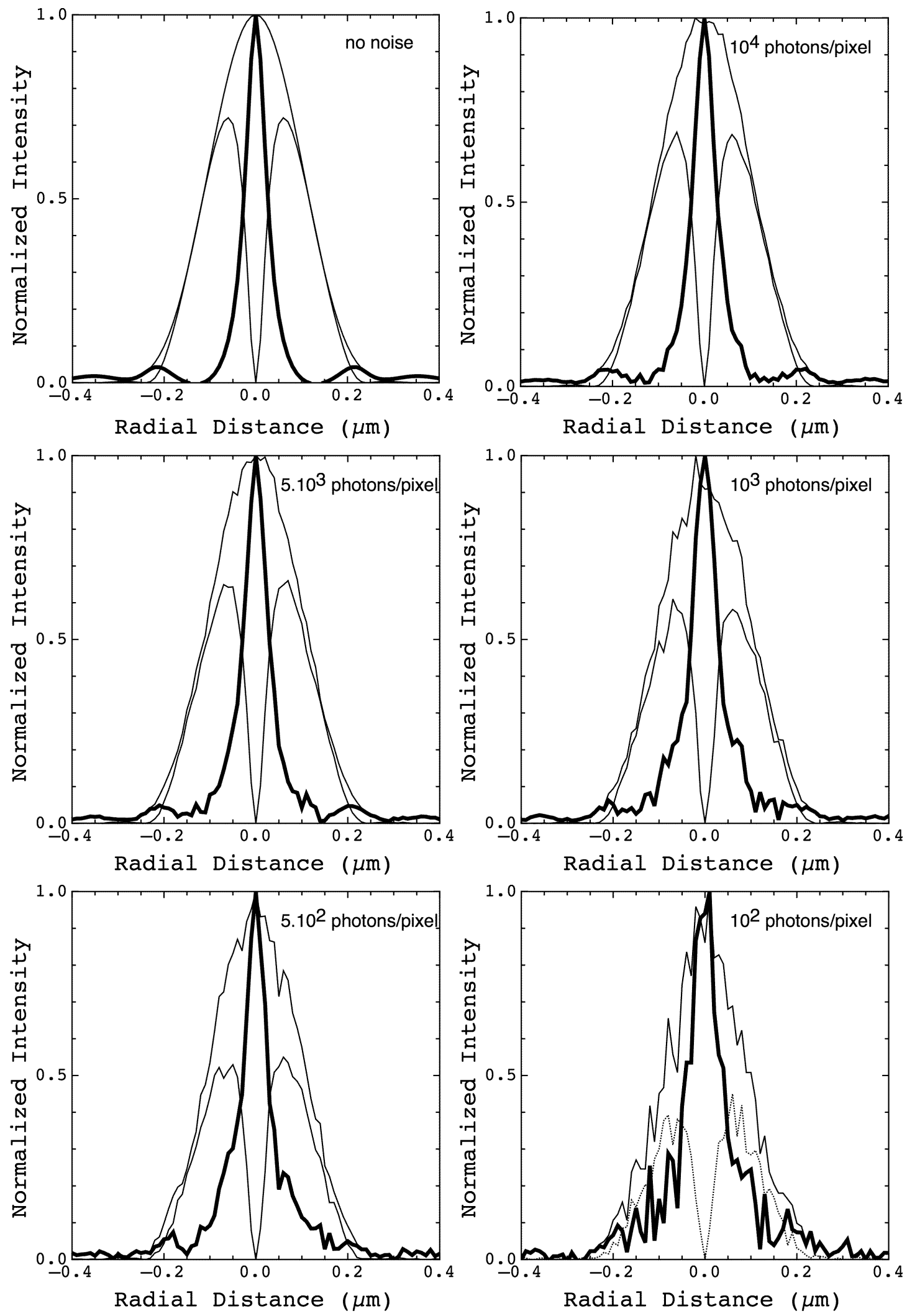
FIGURE 5
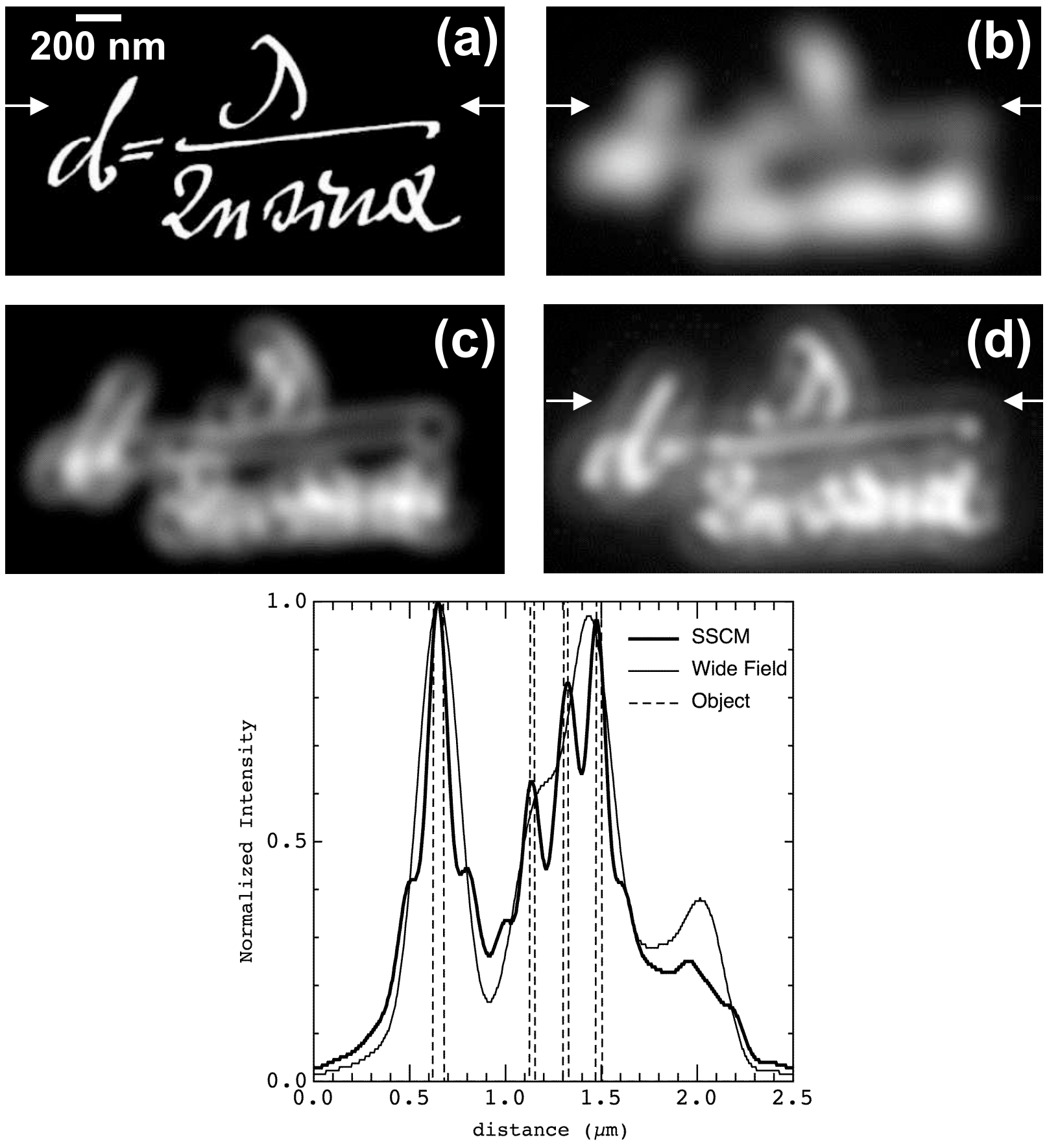